\title{
Results of an International Interlaboratory Trial to Determine Twelve Allergens Using Real-time PCR- and ELISA-based Assays
}

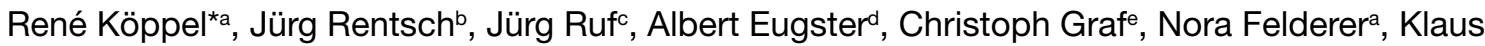 \\ Pietsch', and Evelyn llgg
}

\begin{abstract}
To elucidate the capability of laboratories to determine allergen contents, an international interlaboratory trial was conducted using meat products spiked with 12 allergens. The measurement uncertainty was calculated independent of the applied method simulating realistic situations when comparing analysis certificates from different laboratories. The measurement uncertainty was revealed to be in the best cases $+/-100 \%$, in the worst cases quantification exhibited a measurement uncertainty of higher than $200 \%$ making quantitative analysis impossible. The measurement uncertainty seemed to depend on the analyte and assays used.
\end{abstract}

Keywords: Allergen · Determination · ELISA · Real-time PCR · Sausages

\section{Introduction}

For the wellbeing and safety of persons with allergic reactions, ingredients causing potential allergic reactions must be labeled on each food product. ${ }^{[1,2]}$ Therefore, food control laboratories examine food products regularly for consequent implementation of these regulations by the producers. Currently, the following allergens are listed by the food law of European Union and Switzerland: cereals with gluten, crustacean, egg, fish, milk, mollusks, soy, nuts (almonds, peanuts, cashew, hazelnut, macadamia, walnut, Brazil nut, pecan and pistachio, Queensland nuts), sesame, celery, mustard, lupin and sulphites.

To determine allergens from animal or plant sources, real-time polymerase chain reaction (PCR) and enzyme linked immunoassays (ELISA) are well-recognized methods. ${ }^{[3-13]}$ Both methods are prone to matrix effects like loss of analyte during production and inhibition. ELISA does not need expensive equipment whereas

\footnotetext{
${ }^{*}$ Correspondence: Dr. R. Köppela

Tel.: +41432447224

E-mail: rene.koeppel@klzh.ch

afficial Food Control Authority of the Canton of

Zurich, Zürich, Switzerland,

bswiss Quality Testing Services (SQTS) Courtepin, Switzerland

'Official Food Control Authority of the Canton

Thurgau, Frauenfeld, Switzerland

'Cantonal Office of Consumer Protection Aargau, Aarau, Switzerland

eCantonal Office of Consumer Protection Bern, Switzerland

fState Institute of Chemical and Veterinarian Analysis Freiburg, Germany

${ }^{9}$ Cantonal Food Control Authority of Basel,

Switzerland
}

PCR needs a real-time thermocycler. Run in multiplex format, PCR may have an advantage when analyzing mainly unknown samples.

For most allergens no certified reference material is available. In addition, only little information about stability and range of allergen contents which are quantifiable is available. Many studies, such as interlaboratory trials or proficiency studies, address these questions for only a few allergens. This interlaboratory trial tried to gain an overview for meat products like raw and boiled sausages for 12 allergens at once, independent of the applied method.

To overcome the lack of certified reference material, both reference and sample material was produced and provided for the calibration of measurement of all participants.
We decided to make our own meat products because commercial products often contain undeclared allergens. Usually boiled meat products are of finer texture than raw matured products and therefore would be more homogenous and suitable as reference materials. To assess the contribution of homogeneity to the measurement uncertainty both rough cut matured and fine textured boiled meat-products were produced. A set of boiled and a set of raw matured reference sausages were produced from the same starting material (see Table 1) as the unknown samples. These reference sausages were used for the calibration of the assays. Three of the unknown samples belonged to the matured product group (Cevapcici, Landjäger and Salami) and one represented a boiled product (Sucuk). In addition and prior to this in-

Table 1. Reference sausages and samples. Recipe for $100 \mathrm{~kg}$ of reference sausage (type Landjäger, raw sausages and type boiled sausages) and sample sausage used in this study. Values are given in $\mathrm{kg}$ for the production of $100 \mathrm{~kg}$, taking reduction of the weight during the production process in account.

\begin{tabular}{|l|c|c|c|c|c|c|c|c|c|}
\hline $\begin{array}{l}\text { Fraction } \\
\text { \% }\end{array}$ & $\begin{array}{c}\text { Kal A } \\
\text { LJ }\end{array}$ & $\begin{array}{c}\text { Kal B } \\
\text { LJ }\end{array}$ & $\begin{array}{c}\text { Kal } \\
\text { C LJ }\end{array}$ & $\begin{array}{c}\text { Kal } \\
\text { D LJ }\end{array}$ & $\begin{array}{c}\text { Kal E } \\
\text { LJ }\end{array}$ & $\begin{array}{c}\text { Cevap- } \\
\text { cicci }\end{array}$ & $\begin{array}{c}\text { Land- } \\
\text { jäger }\end{array}$ & Salami & Sucuk \\
\hline Beef & 1 & 8 & 22 & 31 & 48 & 47.6 & 23.2 & 5 & 42 \\
\hline Pork & 31 & 48 & 22 & 8 & 1 & 14.3 & & 48 & 3 \\
\hline Horse & 48 & 31 & 22 & 1 & 9 & 9.5 & 27.8 & 45 & 3 \\
\hline Sheep & 8 & 1 & 22 & 48 & 31 & 23.8 & 1.85 & 2 & 28 \\
\hline Lard (pork) & & & & & & & 39.8 & & \\
\hline Water / ice & 2.4 & 2.4 & 2.4 & 2.4 & 2.4 & 2.4 & & & \\
\hline Curing salt & 2.44 & 2.44 & 2.44 & 2.44 & 2.44 & 0.1 & 2.32 & & 20 \\
\hline $\begin{array}{l}\text { Aller- } \\
\text { gens and }\end{array}$ & 10 & 10 & 10 & 10 & 10 & 2.4 & 5.05 & & 4 \\
additives & & & & & & & & & \\
\hline
\end{tabular}


terlaboratory trial, PCR methods were provided and established at the participating laboratories, if required. Finally, results for the four unknown samples for all 12 allergens had to be generated.

The results represent an overview of the actual capabilities of ELISA- and PCRbased methods for the determination of 12 allergens in sausages.

\section{Materials and Methods}

\subsection{Allergens Used for Spiking of the Reference Sausages and Sample Sausages}

Nuts: The following allergenic nuts were used as spiking material: Peanut, hazelnut, almond, sesame, walnut, pistachio and cashew: Whole nuts and seeds were purchased from a local retailer. Milling led to big particles (due to the high fat concentration) resulting in erratic spiking effects. To solve this problem olive oil was added to transform the nut paste into a slurry. The slurry was filtered through a tea sieve to remove big particles and the proportion of nuts/seeds was determined in the slurry. This slurry was used for spiking.

Soya: Partially defatted soya-flour from Hensel Schoeneberg $\mathrm{GmbH} \& \mathrm{Co}$, 71106 Megstadt, Germany was used.

Celery: Celery bulbs were purchased from a local retailer. After cutting into pieces they were dried overnight at $80{ }^{\circ} \mathrm{C}$ and milled to a fine powder.

Egg: Whole egg powder from Lüchinger Schmied, CH 8302 Kloten, Switzerland Art. Nr 36051 was used.

Mustard: Commercially available 'Coleman's mustard' was used. This product was chosen because of its long tradition (since 1814) and worldwide distribution. It is a very fine powder of yellow and brown mustard.

Lupin: Toasted lupin powder 'FraluT' from FA L.I. Frank, Oude Rijkstraat 32-40 7391 Me Twello, Netherlands

In total 12 allergens were spiked. Milk and gluten were neither spiked nor determined. The spiking level was between 360 ppm and $4237 \mathrm{ppm}$. This range is at least 10 times above the detection level and therefore suitable to be quantified.

\subsection{Reference and Sample \\ Sausages}

The production process and meat composition are briefly described in Table 1 . The precise production process of the reference and sample sausages was described in a previous publication. ${ }^{[14]}$ Calibration curves were prepared from at least four reference points to exclude accidental correlation. Five were chosen to be able to skip one measurement point without compromising the measurement. Cevapcicci and
Landjäger samples were produced by the same butchers that produced the calibration sausages. Salami and Sucuk samples were produced by two other butchers using the identical spiking material. The amount of spiking material was chosen to ensure that only results were gained within the quantification range, excluding false positive or false negative results. Therefore we choose spiking levels between 32 and 3200 ppm $(\mathrm{mg} / \mathrm{kg})$.

\subsection{DNA Isolation and PCR}

Each laboratory applied its own DNA isolation method. To compensate for different isolation efficiencies, all participants were asked to determine the concentration of the DNA photospectrometrically after isolation and to use $100 \mathrm{ng}$ DNA in total as template for the PCR.

Two tetraplex real-time PCR-systems

(AllAllA and AllAllB) determining DNA contents of peanut, soya, celery, hazelnut, beef, almond, egg, sesame, walnut, pistachio, cashew, white and black mustard and lupin were published earlier ${ }^{[15,16]}$ and applied where no other systems were available in the individual laboratory.

For the allergens walnut, ${ }^{[17]}$ cashew, ${ }^{[18]}$ and mustard, ${ }^{[19]}$ published single PCR systems and a new designed system for pistachio were combined to a multiplex real-time PCR-system, called AllAllE. After validation this system was provided to the participants if required (for details see Table 2). AllAllE exhibited a sensitivity of $0.032 \mathrm{ppm}$ or better and a rSD of $32 \%$ or better for all analytes. Beside these PCR-systems different in-house systems were applied and one laboratory applied a kit from Congen to determine the content of almond.

Table 2. Multiplex real-time PCR-System AllAllE for the simultaneous detection of walnut, pistachio, cashew and mustard (Sinapsis Alba and Brassicacea).

\begin{tabular}{|l|l|l|}
$\begin{array}{l}\text { Primer/ } \\
\text { Probe }\end{array}$ & $\begin{array}{l}\text { Final } \\
\text { conc. } \\
\mu \mathbf{M}\end{array}$ & Sequence \\
\hline Walnut & & \\
\hline Jugl F & 0.5 & GCG CAG AGA AAG CAG AG \\
\hline Jugl R & 0.5 & CTC ATG TCT CGA CCT AAT GCT \\
\hline $\begin{array}{l}\text { Jugl } \\
\text { Fam }\end{array}$ & 0.05 & $\begin{array}{l}\text { ATT GTG CCT CTG TTG CTC CTC } \\
\text { TTC CCG }\end{array}$ \\
\hline
\end{tabular}

Pistachio

\begin{tabular}{l|l|l} 
Pis1 F & 0.5 & $\begin{array}{l}\text { CCA AGG TGA TCA ACA TGG } \\
\text { ACA GG }\end{array}$
\end{tabular}

\begin{tabular}{l|l|l} 
Pis1 R & 0.5 & CCT CTT TGT GCT CCC CGT ATT
\end{tabular} $\mathrm{C}$

Pis1 Joe 0.05 AGC AGC ACC ACG GCG AAT ACA GGC

Mustard

CC CAA CYT TGA AAG GAG

CWT CCA AAG C

$\begin{array}{lll}\text { Senn R5 } & 0.1 & \text { C ATG GTC TTC TKG AAG GGA }\end{array}$ CAA ACA CTA ACT TG

Senn F6 0.1 C ATG GTC TTC TTG AAG GGA CAA ACR CTW ACT TG

$\begin{array}{lll}\text { Senn R7 } & 0.1 & \text { C ATG GTC TTC TGG AAG GGR }\end{array}$ CAA ATG CTA ACT TG

$\begin{array}{lll}\text { Senn } 1 & 0.05 & \text { TGC AGC AWG TRA TTA GCC }\end{array}$ Cy5 GTA TCT ACC AGA CYK C

Senn2 0.05 AGC AGC AAA TGG TGA GCC Cy5 GTA TCT ACC AGA CCG C

Cashew

$\begin{array}{lll}\text { Cash2 F } & 0.5 \text { TGC CAG GAG TTG CAG GAA GT }\end{array}$ \begin{tabular}{l|l} 
Cash2 R & 0.5 GCT GCC TCA CCA TTT GCT CTA
\end{tabular}

Cash 0.05

Rox
$67 \mathrm{bp}$

Size

GenBank acc.no. /source/labelling

88bp

AF066055

[4]

FamBHQ1

$77 b p$

Y07600 Pistacia vera this work

Joe/BHQ-1

170- $\quad$ sin A genes e.g. 180bp S54101

[6]

Cy5/BHQ-2

Cy5/BHQ-2

AY081853

[5]

Rox/BHQ2 


\subsection{ELISA}

Five laboratories applied ELISA kits from the following producers: Ridascreen (egg, hazelnut, lupin, peanut, almond), R-Biopharm (mustard, sesame, soya, almond), Neogen (egg, hazelnut), Tepnel (egg, peanut, sesame, soya), Transia (hazelnut, lupin), BioKits (walnut), Veratox (mustard)

\section{Results and Discussion}

Measurements were performed by each laboratory individually and in accordance with routine procedures. All data presented in this study are expressed in $\mathrm{ppm}(\mathrm{mg} / \mathrm{kg})$ of allergen.

Twenty-one data sets were produced by eighteen laboratories from Switzerland and Germany. No data set was excluded.

Table 3. Compilation of the results for Cevapcicci: Relative standard deviation (rSD) and measurement uncertainty were calculated from all datasets. Measurement uncertainty was extended with the factor 2 leading to a probability for a realisation of $95 \%$ interval for one data point. The reason for a high MU was estimated to be combinations of high rSD (rSD) and/or false calibration (cal).

\begin{tabular}{|l|r|r|}
\hline $\begin{array}{l}\text { Cevapcicci } \\
\text { Measured mean } \\
\text { value }\end{array}$ & Hazelnut & Lupine \\
\hline True value & 1400 & 537 \\
\hline rSD \% & 163 & 54 \\
\hline MU(ext) rel. \% & $\mathbf{3 2 7}$ & $\mathbf{2 7 0}$ \\
\hline $\begin{array}{l}\text { Explanation } \\
\text { for high MU }\end{array}$ & rSD & cal \\
\hline
\end{tabular}

In total, roughly 10,000 data points were collected (not presented). All participants used the standard reference sausages with known concentrations of the allergens (KLJ and $\mathrm{KBW}$ ) for the calibration of their assays. The concentrations of allergens in the sample sausages were kept unknown for the participants. Unusually to many proficiency trials applying ELISA methods the results were not grouped according to the test-kits used and the true value (spike) was taken to calculate the accuracy. Therefore a realistic measurement uncertainty could be calculated giving a realistic impression of repeatability including the interlaboratory variation.

The results indicated that lowest measurement uncertainties were gained by using calibration sausages of the boiled type (fine texture). Therefore only results gained by this type of calibration are presented here. We compiled the measurement uncertainties (MU) calculated by geometrical addition of $\mathrm{rSD}$ and relative deviation from the true value extended by a factor 2 in Tables 3 to 6 .

Reasons for high MU were assessed for each allergen and sample combination. The reasons for insufficient results may be divided in two groups: high systematically deviation between the calibration sausages and the measured values (false calibration, cal). Possible explanations may include e.g. different production process, different material (different sub-species) or different storage conditions (e.g. $\mathrm{pH}$, fermentation). Another group exhibited a high variation of the results due to e.g. inhomogeneity of the sample and/or the reference material and/or high intrinsic variation of the assays.

As all sausages (unknown samples and calibration sausages) were spiked with the same material different sub-species as a reason for high systematic deviation can be excluded. Inhomogeneity can also be excluded if the result for at least one allergen was acceptable in the sample sausages. This was the case for Landjäger (pistachio MU 102\%), for Salami (walnut MU 101\%) and for Sucuk (sesame MU 120\%). For the Cevapcicci sample this was not observed and high inhomogeneity cannot be excluded as a reason for the high measurement uncertainty.

\subsection{Results According to Sample Sausages (Tables 3 to 6)}

Setting the limit of acceptance was done arbitrary. For acceptance (accept) the

Table 4. Compilation of the results for Landjäger.

\begin{tabular}{|l|c|c|c|c|c|}
\hline Landjäger & Celery & Sesame & Walnut & Pistachio & Mustard \\
\hline Measured mean value & 592 & 371 & 1005 & 538 & 249 \\
\hline True value & 1400 & 500 & 1800 & 690 & 1400 \\
\hline rSD \% & 54 & 41 & 38 & 42 & 84 \\
\hline MU(ext) rel. \% & $\mathbf{2 9 4}$ & $\mathbf{1 0 8}$ & $\mathbf{1 7 5}$ & $\mathbf{1 0 2}$ & $\mathbf{9 4 0}$ \\
\hline Explanation for high MU & cal & accept & cal & accept & cal + rSD \\
\hline
\end{tabular}

Table 5. Compilation of the results for Salami.

\begin{tabular}{|c|c|c|c|c|c|c|c|c|c|c|c|}
\hline Salami & Peanut & Soya & Celery & Almond & Egg & Sesame & Walnut & Pistachio & Cashew & Mustard & Lupin \\
\hline Measured mean value & 540 & 2371 & 1295 & 918 & 552 & 925 & 2310 & 1652 & 834 & 971 & 2724 \\
\hline True value & 360 & 4556 & 805 & 597 & 1695 & 1599 & 1695 & 1556 & 4556 & 1243 & 932 \\
\hline $\mathrm{rSD} \%$ & 67 & 71 & 156 & 50 & 41 & 53 & 43 & 105 & 43 & 79 & 126 \\
\hline MU(ext) rel. \% & 150 & 233 & 321 & 122 & 422 & 180 & 101 & 210 & 897 & 168 & 283 \\
\hline $\begin{array}{l}\text { Explanation } \\
\text { for high MU }\end{array}$ & $\begin{array}{l}\mathrm{cal}+ \\
\mathrm{rSD}\end{array}$ & $\begin{array}{c}\mathrm{cal}+ \\
\mathrm{rSD}\end{array}$ & $\mathrm{rSD}$ & accept & cal & cal & accept & $\mathrm{rSD}$ & cal & $\begin{array}{c}\mathrm{cal}+ \\
\mathrm{rSD}\end{array}$ & $\begin{array}{l}\mathrm{cal}+ \\
\mathrm{rSD}\end{array}$ \\
\hline
\end{tabular}

Table 6. Compilation of the results for Sucuk.

\begin{tabular}{|c|c|c|c|c|c|c|c|c|c|c|}
\hline Sucuk & Peanut & Celery & Hazelnut & Almond & Egg & Sesame & Walnut & Pistachio & Mustard & Lupin \\
\hline Measured mean value & 1994 & 871 & 315 & 3598 & 1361 & 1054 & 6366 & 1807 & 196 & 6439 \\
\hline True value & 1182 & 554 & 386 & 1932 & 1438 & 1438 & 1438 & 1010 & 190 & 4237 \\
\hline $\mathrm{rSD} \%$ & 69 & 90 & 66 & 80 & 103 & 47 & 92 & 73 & 63 & 181 \\
\hline MU(ext) rel. \% & 160 & 195 & 139 & 185 & 207 & 120 & 240 & 171 & 126 & 372 \\
\hline $\begin{array}{l}\text { Explanation } \\
\text { for high MU }\end{array}$ & $\begin{array}{l}\mathrm{cal}+ \\
\mathrm{rSD}\end{array}$ & rSD & accept & $\begin{array}{l}\mathrm{cal}+ \\
\mathrm{rSD}\end{array}$ & rSD & accept & $\begin{array}{l}\mathrm{cal}+ \\
\mathrm{rSD}\end{array}$ & $\begin{array}{c}\mathrm{cal}+ \\
\mathrm{rSD}\end{array}$ & accept & rSD \\
\hline
\end{tabular}


MU had to be below $150 \%$ focusing on a single sample. Considering all samples the limit was extended to $200 \%$.

Cevapcicci: The results (Table 3) exhibited a measurement uncertainty (MU) of $327 \%$ for hazelnuts. This percentage is high and estimated to be due to the bad precision of the applied method (rSD 163\%). The mean value corresponded well with the true value.

The results for lupin also exhibited a high measurement uncertainty of $270 \%$ but this was mainly based on false calibration. As all reasons for systematically deviations are expected to affect hazelnut and lupin analytes similarly, the reason for this deviation remains unclear.

Landjäger: Considering that both $\mathrm{PCR}$ and ELISA methods were applied, the results for sesame and pistachio were satisfactory with a MU close to $100 \%$. This suggests that precise results can be attained even when analyzing samples with rough texture and different methods.

The results for walnut had an augmented MU possibly due to false calibration. This was also concluded for celery (MU $294 \%$, see Table 4). The results for mustard were simply false (MU 940\%) and incomparable, possibly mainly due to false calibration (reason unclear). But also the rSD was high (rSD 84\%).

Salami: The results for almond and walnut exhibited an acceptable MU (see Table 5). Results for cashew and egg were not reproducible with a MU of $422 \%$ and $897 \%$. The results for the other allergens were placed in the middle field between $150 \%$ and $321 \%$. The reason for insufficient results for cashew is highly possibly a consequence of the cross reactivity of the AllAllE between cashew and pistachio (also spiked in this sample).

The amount of egg protein was exclusively determined with ELISA assays. A high deviation based on different calibration material can be excluded as the calibration sausages contained the same material as the unknown samples (see results for egg in Sucuk). It seems that all applied ELISA-Kits were affected by systematic deviation which may origin from matrix effects.

Sucuk: Results exhibited an acceptable (see Table 6) MU for hazelnut, sesame and mustard. Lupin exhibited an unacceptable
MU of $372 \%$, possibly based in a false calibration in conjunction with a high deviation of $181 \%$. The Salami sample had already exhibited a high rSD for lupin. Therefore it seems that the applied methods intrinsically exhibit a high rSD. The method for the determination of lupin should be ameliorated to reduce the high rSD of results. The other analytes exhibited a MU between $160 \%$ and $240 \%$, often based in a high rSD.

\subsection{Results According to Allergens (Table 7)}

Peanut, almond, sesame: The results for these allergens were generated by ELISA and PCR methods and reached an acceptable MU.

Walnut, pistachio: The results for these allergens were generated by PCR methods only and reached an acceptable MU.

Soya: The results for soya were generated by ELISA and PCR and exhibited a MU above $200 \%$, which was decided to be unsatisfactory. Systematic deviation and high rSD led to the high MU. Lupin (taxonomically close to soya) was also used as spike. But the results underestimated the true value, in consequence this potentially cross reactive addition can be excluded as reason for the high deviation. Therefore the reason for the systematic deviation remains unclear. However, reducing the deviation of the assays should ameliorate the MU.

Celery and hazelnut: Mainly high rSD led to the high MU of $270 \%$ and $233 \%$. Development of more consistent methods seems to be advisable for the determination of these two allergens.

Egg: To detect egg, which contains very low amounts of DNA, PCR methods are not appropriate. Therefore the results for egg were generated using ELISA only. Four different kits were applied. Obviously these kits produced inconsistent results as already discussed above.

Cashew: The results for cashew were false. This may be the result of the known cross reactivity produced by AllAllE in conjunction with pistachio. More specific PCR systems have to be designed.

Mustard and lupin: The high MU seems to be mainly based on systematic deviation. Mustard includes different Brassicaceae and Sinapsis species. There are also several different subspecies of lupin in use. For quantification choosing the corresponding calibrator is crucial but will remain impossible for unknown samples. However, in our study reference material and spike were the same. In consequence, this reason can be excluded in this interlaboratory trial and therefore the reason for the high MU remains unclear.

\section{Conclusion}

Often a MU of $30 \%$ can be expected for single measurements in the same laboratory using e.g. real-time PCR (without extension factor 2). Including the extension factor and different laboratories using different methods, a MU of $100 \%$ may be realistic. Best results were gained when measuring walnut in the rough textured Salami sample (MU 101\%). These results may define the benchmark in this interlaboratory trial which seems to be achievable independent of the applied method (ELISA or PCR) and texture of the product.

For peanut, almond, sesame, walnut, and pistachio, reproducible results may be generated when using the same reference material for calibration. Surprisingly, this may even be the case using combinations of ELISA and PCR methods. The methods for egg (only ELISA), celery, hazelnut, cashew, soya, mustard, and lupin must be ameliorated. Their results exhibited an unacceptably high measurement uncertainty. The reason for the systematic deviation was not uncovered during this interlaboratory trial.

As allergens are determined absolute, the definition of reference material is crucial. This is a prerequisite for all actual and future methods e.g. mass spectrometrically. ${ }^{[20]}$ But at the moment no certified reference material of broad acceptance is available. Such material would have to be designated, produced and accepted by main laboratories and kit producers which is not yet the case.

Often proficiency trials do not calculate accuracy and group results according to the kit manufacturer. This may have led in the past to an over-optimistic perception of achievable measurement uncertainty. This does not help development of meth-

Table 7. Compilation of the overall performance according to the allergen determined. It was calculated by averaging the MU for all samples. For acceptance the MU had to be below $200 \%$ or the probably main reason for high MU was estimated (rSD/cal).

\begin{tabular}{|c|c|c|c|c|c|c|c|c|c|c|c|c|}
\hline & Peanut & Soya & Celery & Hazelnut & Almond & Egg & Sesame & Walnut & Pistachio & Cashew & Mustard & Lupin \\
\hline $\begin{array}{l}\text { Mean value } \\
\text { of } \mathrm{MU}\end{array}$ & 155 & 233 & 270 & 233 & 154 & 315 & 136 & 172 & 161 & 897 & 411 & 308 \\
\hline $\begin{array}{l}\text { Explanation } \\
\text { for high MU }\end{array}$ & accept & $\begin{array}{c}\mathrm{cal}+ \\
\mathrm{rSD}\end{array}$ & $\mathrm{rSD}$ & $\mathrm{rSD}$ & accept & $\begin{array}{c}\mathrm{cal}+ \\
\mathrm{rSD}\end{array}$ & accept & accept & accept & cal & ok cal & cal \\
\hline
\end{tabular}


ods with adequate interlaboratory accuracy and should be stopped.

In summary, comparable and reproducible quantitative results can only be produced in cases where the applied methods exhibit an acceptable accuracy and precision in conjunction with commonly applied reference material. With ameliorated methods (e.g. using multi-copy genes) and commonly accepted certified reference material this goal was shown here to be achievable in principle for all allergens. At the moment quantitative measurements of allergens have to be interpreted with care and in context of the production process. Comparability and reproducibility have not yet been achieved. However, the actual methods remain important tools to control and enhance product security for persons with allergic reactions.

\section{Acknowledgements}

We would like to thank all the participating laboratories: Swiss Cantonal Laboratories of Aargau (AVS AG), Bern, Basel Stadt, Zürich, Geneva, Swiss Quality Testing Services (SQTS), Central Laboratory Coop, Biolytix, Biosmart GmbH, Eurofins Hamburg, CVUA Freiburg, CVUA Sigmaringen, CVUAMelingen, LGL Bayern Oberschleissheim, LGL Bayern Erlangen, TLLV Thüringen, R-Biopharm Darmstadt, GALAB GmbH Geesthacht.

Special thank goes to the SQTS, AVS AG and the Cantonal Laboratory Zürich for the production and quality control of reference and sample sausages.
[1] Swiss Food Law: Art. 8 Abs. 1 der Verordnung über die Kennzeichnung und Anpreisung von Lebensmitteln vom 23.11.2005.

[2] Directive 2003/89EC of the European Parliament and Council and its Annex IIIa.

[3] C. Hupfer, H. Waiblinger, U. Busch, Eur. Food Res. Technol. 2007, 225, 321.

[4] S. Rossi, E. Scaravelli, A. Germini, R. Corradini, C. Fogher, R. Marchelli, Eur. Food Res. Technol. 2006, 223, 1.

[5] L. Dovičovičová, L. Olexova, D. Pangallo, P. Siekel, Eur. Food Res. Technol. 2004, $218,493$.

[6] R. Poms, E. Anklam, J. AOAC Int. 2004, 87, 1391.

[7] O. Stephan, S. Vieths, J. Agric. Food. Chem. 2004, 52, 3754.

8] H. Hird, J. Lloyd, R. Goodier, J. Brown, P Reece, Eur. Food Res. Technol. 2003, 217, 265.

[9] L. Herman, J. De Block, R. Viane, Food Sci. Technol. 2003, 38, 633

[10] T. Holzhauser, O. Stephan, S. Vieths, J. Agric. Food Chem. 2002, 50, 5808.

[11] Official collection of methods according to $\S 64$ of the German food and feed law (2008):
Detection of a DNA sequence from celery in sausages by means of real-time PCR. Method L 08.00-56 (12/2008).

[12] A. Ehlert, A. Demmel, C. Hupfer, U. Busch, K. H. Engel, Food Addit. Contamin. 2009, 26, 409.

[13] 'Detecting allergens in food', Ed. S. Koppelman, formerly TNO Nutrition and Food Research, The Netherlands and S. Hefle, formerly Food Allergy Research and Resource Program, USA) Woodhead Publishing Series in Food Science, Technology and Nutrition No. 117.

[14] R. Köppel, A. Eugster, J. Ruf, J. Rentsch, J. AOAC Int. 2012, 95, 494.

[15] A. Demmel, C. Hupfer, E. Ilg, U. Busch, K. H. Engel, J. Agric. Food Chem. 2008, 56, 4328.

[16] R. Köppel, V. Dvorak, F. Zimmerli, A Breitenmoser, A. Eugster, H. Waiblinger, Eur. Food Res. Technol. 2010, 230, 367.

[17] B. Brezna, L. Hudecova, T. Kuchta, Eur. Food Res. Technol. 2006, 223, 373.

[18] J. L. Brzezinski, J. AOAC Int. 2006, 89, 103.

[19] S. Mustorp, U. Engdahl-Axelsson, U. Svensson, Eur. Food Res. Technol. 2007, 226, 771.

[20] J. Heick, M. Fischer, S. Kerbach, U. Tamm, B. Popping, J. AOAC Int. 2011, 94, 1060. 